\title{
Combinatorics of the 2-species exclusion processes, marked Laguerre histories, and partially signed permutations
}

\author{
Sylvie Corteel \\ Department of Mathematics \\ UC Berkeley, Berkeley CA, U.S.A \\ corteel@berkeley.edu
}

\author{
Arthur Nunge \\ Fakultät für Mathematik \\ Universität Wien, Austria \\ arthur.nunge@univie.ac.at
}

Submitted: Jul 30, 2019; Accepted: May 25, 2020; Published: Jun 26, 2020

(C) The authors. Released under the CC BY-ND license (International 4.0).

\begin{abstract}
Starting from the two-species partially asymmetric simple exclusion process, we study a subclass of signed permutations, the partially signed permutations, using the combinatorics of Laguerre histories. From this physical and bijective point of view, we obtain a natural descent statistic on partially signed permutations; as well as partially signed permutations patterns.
\end{abstract}

Mathematics Subject Classifications: 05A05, 05A19

\section{Introduction}

The two-species partially asymmetric simple exclusion process (2-PASEP) is a Markov chain with two types of particles $(\bullet$ and $\bullet$ ) and holes (०). Particles can hop to the right and to the left and particles of type $\bullet$ can enter and exit the system. If there are no particles of type $\bullet$, we recover the classical PASEP. See Section 3 and [27] for a detailed definition. The classical PASEP has given rise to beautiful combinatorics related to Laguerre histories [16], permutations $[5,16,25]$, permutation tableaux $[8,7,25]$, alternative tableaux [20] and staircase tableaux [9] in its most general case. All these objects are shown to be connected to the PASEP thanks to the Matrix Ansatz [12] and the fact that the partition function of the model is related to the moments of the Askey Wilson polynomials [28]. In special cases, we can define a Markov chain on the permutations or the tableaux that projects to the ASEP chain. See [7] for example. This is classical in Markov chain theory and is called lumpability $[17,3]$.

In the case of the 2-PASEP the Matrix Ansatz extends naturally [27]. We shall detail this in Section 3. The partition function is now related to the mixed moments of the Askey Wilson polynomials [10]. It is therefore expected (but not at all trivial) to see that 
the combinatorics extends to this generalized model. The alternative tableaux become rhombic alternative tableaux (RATS) [18] and the permutations become assemblées of permutations [19]. In the most general case we get rhombic staircase tableaux [6].

In this paper we take a slightly different approach, as some of the statistics coming from the 2-PASEP are not natural on the assemblées of permutations. We generalize the results of $[5,16]$ related to Laguerre histories and permutations. In these cases the states of the PASEP are in bijection with compositions and the statistic coming from the PASEP is in bijection with the weight of the paths or equivalently the number of the generalized patterns $(31-2)$ of the permutation. Generalized patterns were first introduced in a general framework by Babson and Steingrímsson in [1], but some instances had been treated previously in various contexts. For example, the pattern $(31-2)$ is implicit in [13] and in [24]. We define some generalization of Laguerre histories : the marked Laguerre histories. We give a bijection between the marked Laguerre histories and a subclass of signed permutations where we do not put a sign on 1 . We call them partially signed permutations. For example, the partially signed permutations of size 2 are $12,21,1 \overline{2}$ and $\overline{2} 1$. The states of the 2-PASEP are in bijection with segmented compositions and the statistic coming from the 2-PASEP is in bijection with the weight of the paths or equivalently the number of some generalized patterns of the partially signed permutation.

All the detailed definitions are given in Section 2. In Section 3, we give a new solution for the interpretation of the probabilities of the 2-PASEP. In Section 4, we use this solution to interpret these probabilities using marked Laguerre histories. In Section 5, we use the Françon-Viennot bijection to obtain an interpretation in terms of generalized permutations. In Section 6, we give another interpretation in terms of large Laguerre histories and give an explicit involution on these large Laguerre histories that explains the so-called particle hole symmetry of the process. We end the paper with some final comments and open problems in Section 7.

\section{Notations and definitions}

\subsection{Signed permutations and segmented compositions}

A signed permutation $\boldsymbol{\sigma}$ of size $n$ is a permutation of $n$ such that each value has a sign plus or minus. We denote by $B_{n}$ the set of signed permutations of size $n$. We overline negative values and we say that $\bar{k} \in \boldsymbol{\sigma}$ if the value $k$ has a negative sign in $\boldsymbol{\sigma}$. For example, $\boldsymbol{\sigma}=\overline{2} 57836 \overline{4} 1$ is a signed permutation of size 8 .

When we compare two values $\boldsymbol{\sigma}_{i}$ and $\boldsymbol{\sigma}_{j}$ of a signed permutation $\boldsymbol{\sigma}$, we use the order $\overline{1}<1<\overline{2}<2<\cdots$.

Definition 1. A partially signed permutation is a signed permutation where 1 is not signed. We denote by $B_{n}^{\prime}$ the set of these permutations.

The overlined values of a partially signed permutation are its negative values. We denote by $\operatorname{Sign}(\boldsymbol{\sigma})$ the set of all overlined values of a partially signed permutation $\boldsymbol{\sigma}$.

For example, $\boldsymbol{\sigma}=\overline{2} 57836 \overline{4} 1$ is an element of $B_{n}^{\prime}$ and its set of overlined values is $\operatorname{Sign}(\overline{2} 57836 \overline{4} 1)=\{2,4\}$. 
We define generalized patterns [1] for partially signed permutations.

Definition 2. A 31-2 pattern of a partially signed permutation $\boldsymbol{\sigma}$ is a pair $\left(\sigma_{i} \sigma_{i+1}, \sigma_{j}\right)$ such that $j>i+1$ and $\boldsymbol{\sigma}_{i}>\boldsymbol{\sigma}_{j}>\boldsymbol{\sigma}_{i+1}$. We denote this pattern by $\boldsymbol{\sigma}_{i} \boldsymbol{\sigma}_{i+1}-\boldsymbol{\sigma}_{j}$.

A $(31, \overline{2})$ pattern is a pair $\left(\sigma_{i} \sigma_{i+1}, \bar{k}\right)$ such that $\bar{k} \in \boldsymbol{\sigma}$ and $\boldsymbol{\sigma}_{i} \geqslant \bar{k}>\boldsymbol{\sigma}_{i+1}$.

We denote by $\operatorname{tw}(\boldsymbol{\sigma})$ the number of $31-2$ patterns of $\boldsymbol{\sigma}$ plus its number of $(31, \overline{2})$ patterns

Note that in the second case the value $\bar{k}$ can be to the left or to the right of $\sigma_{i}$. For example, the 31-2 patterns of $\boldsymbol{\sigma}=\overline{2} 57836 \overline{4} 1$ are $83-6$ and $83-\overline{4}$ and the $(31, \overline{2})$ patterns of $\boldsymbol{\sigma}$ are $(83, \overline{4}),(\overline{4} 1, \overline{2})$, and $(\overline{4} 1, \overline{4})$.

Definition 3. A segmented composition of an integer $n$ is a finite sequence $\mathbf{I}$ of $\ell$ positive integers $\left(i_{1}, \ldots, i_{\ell}\right)$ that sum to $n$ separated by vertical bars or commas.

The descent set of $\mathbf{I}$ (denoted by $\operatorname{Des}(\mathbf{I})$ ) is the set of values $i_{1}+i_{2}+\cdots+i_{k}$ where $i_{k}$ is followed by a comma in $\mathbf{I}$. Similarly, the segmentation set of $\mathbf{I}$ (denoted by $\operatorname{Seg}(\mathbf{I})$ ) is the set of values $i_{1}+i_{2}+\cdots+i_{k}$ such that $i_{k}$ is followed by a bar in $\mathbf{I}$.

For example,

$$
(\operatorname{Des}(1|2| 1,2,2), \operatorname{Seg}(1|2| 1,2,2))=(\{4,6\},\{1,3\}) .
$$

The ADE-word associated with $\mathbf{I}$ is the word $w$ of size $n-1$ such that

$$
\begin{aligned}
i \in \operatorname{Des}(\mathbf{I}) & \Rightarrow w_{i}=E ; \\
i \in \operatorname{Seg}(\mathbf{I}) & \Rightarrow w_{i}=A ; \\
i \notin \operatorname{Des}(\mathbf{I}), i \notin \operatorname{Seg}(\mathbf{I}) & \Rightarrow w_{i}=D .
\end{aligned}
$$

We denote this word by $\operatorname{ADE}(\mathbf{I})$. For example, $\operatorname{ADE}(1|2| 1,2,2)=A D A E D E D$.

Definition 4. The Genocchi descent set of a partially signed permutation $\boldsymbol{\sigma}$ of size $n$ is the set of positive values followed by a smaller value. In other words,

$$
\operatorname{GDes}(\boldsymbol{\sigma}):=\left\{i \mid i \notin \operatorname{Sign}(\boldsymbol{\sigma}), \boldsymbol{\sigma}_{j}=i \Rightarrow \boldsymbol{\sigma}_{j}>\boldsymbol{\sigma}_{j+1}\right\}
$$

The Genocchi composition of descents of a partially signed permutation (denoted by $\mathrm{GC}(\boldsymbol{\sigma}))$ is the segmented composition $\mathbf{I}$ whose descent set is $\{d-1 \mid d \in \operatorname{GDes}(\boldsymbol{\sigma})\}$ and whose segmentation set is $\{s-1 \mid s \in \operatorname{Sign}(\boldsymbol{\sigma})\}$.

Note that if $\boldsymbol{\sigma}$ does not have any overlined values, the statistic GC is the composition of the values of descents minus one and is the same as the one defined in [15]. For example, if $\boldsymbol{\sigma}=\overline{2} 57836 \overline{4} 1$, we have $\operatorname{GDes}(\boldsymbol{\sigma})=\{6,8\}, \operatorname{Sign}(\boldsymbol{\sigma})=\{2,4\}$ so we have $\mathrm{GC}(\boldsymbol{\sigma})=(1|2| 2,2,1)$. 


\subsection{Laguerre histories}

Recall that a Motzkin path of size $n$ is a path going from $(0,0)$ to $(n, 0)$ using increasing steps, decreasing steps, and horizontal steps that never goes below the horizontal axis. For any path $P$, we denote by $P_{i}$ the $i$ th step of $P$. We call the starting (resp. ending) height of a step, the distance between the beginning (resp. end) of this step and the horizontal axis. Unless otherwise specified, the height is understood to be the starting height.

A Laguerre history is a weighted object introduced by Viennot in [29], see also [11]. The Laguerre histories are in bijection with permutations through the Françon-Viennot bijection [14]. These objects have been used to study some properties of the ASEP [16].

Definition 5. A Laguerre history $H$ of size $n$ is a weighted Motzkin path of size $n$ with two different horizontal steps such that

- a $\nearrow$ or $\longrightarrow$ step starting from height $h$ has a weight between 0 and $h$;

- a $\searrow$ or $\rightarrow$ step starting from height $h$ has a weight between 0 and $h-1$.

We denote by $\operatorname{tw}(H)$ the total sum of the weights of $H$.

If $w_{i}$ is the weight of the $i$ th step of a Laguerre history $L$ of size $n$, we call $w=w_{1} \ldots w_{n}$ the weight of $L$.

An example of a Laguerre history of size 8 is given in Figure 1. To avoid cumbersome figures, we only represent the non-zero weights of the steps on the figures.

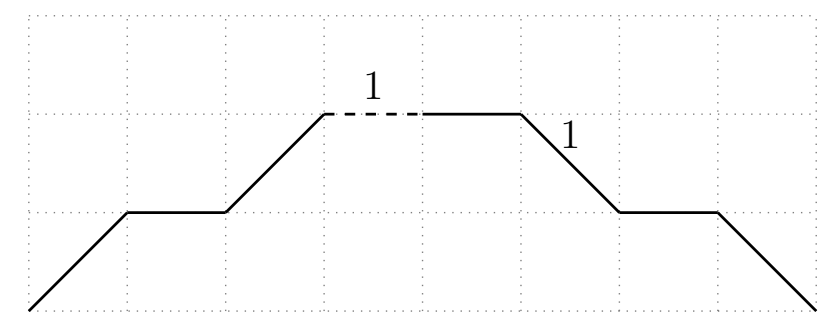

Figure 1: An example of Laguerre history of size 8 and weight 00010100.

The Françon-Viennot bijection [14] is a bijection between Laguerre histories of size $n$ and permutations $\sigma$ in $\mathfrak{S}_{n}$. We denote this map by $\psi_{F V}$. We compare each value of the permutation $\sigma$ with its two neighbors. We use the convention $\sigma_{0}=0$ and $\sigma_{n+1}=n+1$.

Algorithm 6. [14]

- Input: A permutation $\sigma \in \mathfrak{S}_{n}$.

- Output: A Laguerre history $H$ of size $n$.

- Execution: For $k$ from 1 to $n$, let $j$ be such that $\sigma_{j}=k$. The $k^{2}$ th step of $H$ is

$$
-H_{k}=\nearrow \text { if } \sigma_{j} \text { is a valley, i.e., } \boldsymbol{\sigma}_{j-1}>\sigma_{j}<\sigma_{j+1},
$$


$-H_{k}=\searrow$ if $\sigma_{j}$ is a peak, i.e., $\boldsymbol{\sigma}_{j-1}<\sigma_{j}>\sigma_{j+1}$,

$-H_{k}=\longrightarrow$ if $\sigma_{j}$ is a double rise, i.e., $\boldsymbol{\sigma}_{j-1}<\sigma_{j}<\sigma_{j+1}$,

$-H_{k}=-\rightarrow$ if $\sigma_{j}$ is a double descent, i.e., $\boldsymbol{\sigma}_{j-1}>\sigma_{j}>\sigma_{j+1}$.

The weight of $k^{2}$ th step of $H$ is equal to the number of $31-2$ patterns such that $k$ is the number corresponding to 2 in $\sigma$.

The Laguerre history in Figure 1 is the image of the permutation $\sigma=25783641$. Indeed, the valleys of $\sigma$ are 3 and 1; its peaks are 8 and 6 ; its double rises are 2, 5, and 7; and its only double descent is 4 . Finally, its $31-2$ patterns are $83-4$ and $83-6$.

We shall also need the reciprocal map of the Françon-Viennot bijection described by the following algorithm.

Algorithm 7.

- Input: A Laguerre history $H$ of size $n$

- Output: A permutation $\sigma$ of size $n$.

- Initialization: $\sigma=0$;

- Execution: Let $w$ be the weight of $H$. For $k \in\{1, \ldots, n\}$, replace the $\left(w_{k}+1\right)$-st $\circ$ of $\sigma$ by:

$$
\begin{aligned}
& -\circ k \circ \text { if } H_{k}=\nearrow ; \\
& -k \circ \text { if } H_{k}=\longrightarrow ; \\
& -\circ k \text { if } H_{k}=-\rightarrow ; \\
& -k \text { if } H_{k}=\searrow .
\end{aligned}
$$

The final permutation is obtained by removing the last $\circ$.

For example, see (4) for a step by step execution of Algorithm 7 on the Laguerre history of Figure 1.

$$
\begin{gathered}
\sigma=\circ \rightarrow \circ 1 \circ \rightarrow 2 \circ 1 \circ \rightarrow 2 \circ 3 \circ 1 \rightarrow 2 \circ 3 \circ 41 \circ \\
\rightarrow 25 \circ 3 \circ 41 \circ \rightarrow 25 \circ 3641 \circ \rightarrow 257 \circ 3641 \circ \\
\rightarrow 25783641 \circ \rightarrow 25783641
\end{gathered}
$$

\section{The 2-PASEP and the Matrix Ansatz}

The two-species PASEP is a Markov chain whose states are words of length $N$ in the letters $\{\circ, \bullet, \bullet\}$. This was first studied in a more general setting in [27] and then combinatorialy in $[6,10,18,19]$. This Markov chain is described the following way:

Definition 8. Let $q$ be a constant such that $0 \leqslant q \leqslant 1$. The 2-PASEP is the Markov chain on the words in the letters $\circ, \bullet, \bullet$ with transition probabilities: 
- If $x=A \bullet \circ B$ and $y=A \circ \bullet B$ then $P_{x, y}=\frac{1}{N+1}$ (black particle hops right) and $P_{y, x}=\frac{q}{N+1}$ (black particle hops left).

- If $x=A \circ \circ$ and $y=A \circ \circ B$ then $P_{x, y}=\frac{1}{N+1}$ (gray particle hops right) and $P_{y, x}=\frac{q}{N+1}$ (gray particle hops left).

- If $x=A \bullet \bullet B$ and $y=A \bullet \bullet B$ then $P_{x, y}=\frac{1}{N+1}$ (black particle hops right) and $P_{y, x}=\frac{q}{N+1}$ (black particle hops left).

- If $x=\circ B$ and $y=\bullet B$ then $P_{x, y}=\frac{1}{N+1}$ (particle enters from left).

- If $x=B \bullet$ and $y=B \circ$ then $P_{x, y}=\frac{1}{N+1}$ (particle exits to the right).

- Otherwise $P_{x, y}=0$ for $y \neq x$ and $P_{x, x}=1-\sum_{x \neq y} P_{x, y}$.

An example of a chain on three letters among which two are $\bullet$ is given on Figure 2 where we represent the transitions $(N+1) P_{X, Y}$ for $X \neq Y$ and $P_{X, Y} \neq 0$.

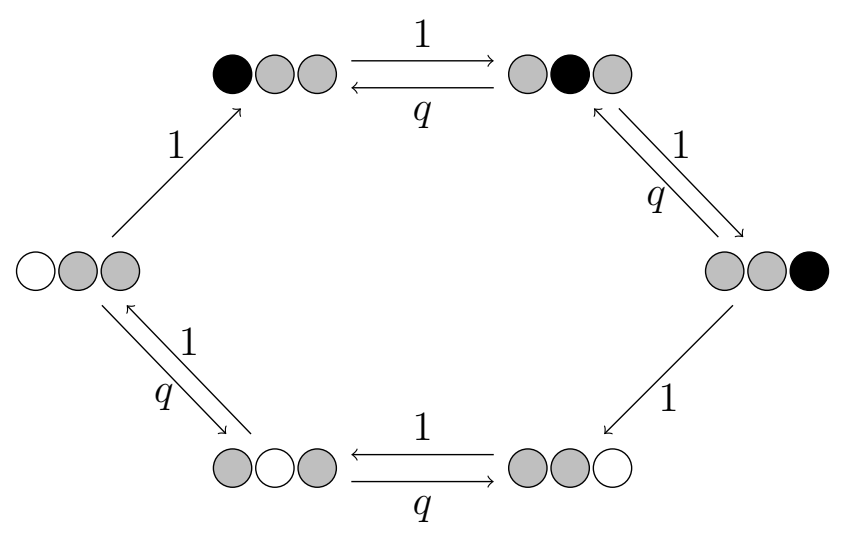

Figure 2: A Markov chain for $N=3$ and $r=2$.

Remark 9. Given two states of the 2-PASEP $x$ and $y$, using the different transitions one can see that the transition from $x$ to $y$ is the same as the one from $\iota(x)$ to $\iota(y)$ where $\iota$ is the map that reverse a state and replace the $\circ$ spots by $\bullet$ and conversely. This is called the particle hole symmetry of the process.

For example, $P_{\bullet}, \bullet, \bullet$ is equal to $P_{\bullet \bullet \circ, \bullet \circ \bullet}$.

To each state $x$ of the 2-PASEP with $N$ sites we associate a word $X(x)$ in $\{A, D, E\}^{N}$ using the following map:

$$
\circ \mapsto E ; \bullet \mapsto D ; \bullet \mapsto A .
$$

We define an involution $\iota$ on words in $\{A, D, E\}^{N}$ that corresponds to the particle hole symmetry.

Definition 10. Let $X \in\{A, D, E\}^{N}$, define $\iota(X)$ as the word obtained after reversing $X$ and replacing $D$ by $E$ and conversely. 
Uchiyama [27] proved that we can use a Matrix Ansatz in order to compute the stationary distribution of the states of the 2-PASEP. We denote by $\operatorname{Prob}(x)$ the stationary distribution of a state $x$.

Proposition 11. [27] Let D, A, E be infinite matrices. Let $\langle W|$ (resp. $|V\rangle)$ be an infinite row (resp. column) vector satisfying the Ansatz:

$$
\begin{aligned}
D E & =q E D+D+E ; \\
D A & =q A D+A ; \\
A E & =q E A+A ; \\
\langle W| E & =\langle W| \\
D|V\rangle & =|V\rangle .
\end{aligned}
$$

Then the probability to be in a state $x$ in $\{\circ, \bullet, \bullet\}^{N}$ with $r$ letters $A$ is:

$$
\operatorname{Prob}(x)=\frac{\langle W|X(x)| V\rangle}{\left[y^{r}\right]\left\langle W\left|(D+y A+E)^{N}\right| V\right\rangle} .
$$

where $\left[y^{r}\right]$ means that we consider the coefficient of the monomial $y^{r}$.

Remark 12. The Ansatz in [27] is more general, corresponding to the 2-ASEP where particles may enter and exit the chain from the right and the left with different rates.

We give here a new solution of this system using the following matrices.

$$
\begin{aligned}
& D=\left(\begin{array}{ccccc}
1 & 1 & 0 & 0 & \ldots \\
0 & {[2]_{q}} & {[2]_{q}} & 0 & \ldots \\
0 & 0 & {[3]_{q}} & {[3]_{q}} & \ldots \\
0 & 0 & 0 & {[4]_{q}} & \cdots \\
\vdots & \vdots & \vdots & \vdots &
\end{array}\right) \\
& E=\left(\begin{array}{ccccc}
0 & 0 & 0 & 0 & \ldots \\
1 & 1 & 0 & 0 & \ldots \\
0 & {[2]_{q}} & {[2]_{q}} & 0 & \ldots \\
0 & 0 & {[3]_{q}} & {[3]_{q}} & \ldots \\
\vdots & \vdots & \vdots & \vdots &
\end{array}\right) \\
& A=\left(\begin{array}{ccccc}
1 & 0 & 0 & 0 & \ldots \\
0 & q & 0 & 0 & \ldots \\
0 & 0 & q^{2} & 0 & \ldots \\
0 & 0 & 0 & q^{3} & \ldots \\
\vdots & \vdots & \vdots & \vdots &
\end{array}\right)(D+E) ; \\
& \langle W|=(1,1,0,0, \ldots) ;
\end{aligned}
$$




$$
|V\rangle=\left(\begin{array}{c}
1 \\
0 \\
0 \\
\vdots
\end{array}\right) .
$$

Lemma 13. The previous matrices and vectors satisfy the equations from Proposition 11. Proof. Let $M=D E$. For all $i, j$, we have

$$
\begin{aligned}
M_{i j} & =\sum_{k=1}^{\infty} D_{i k} E_{k j} \\
& =\sum_{k=i}^{i+1}[i]_{q} E_{k j} .
\end{aligned}
$$

Hence, $M$ is described as follow :

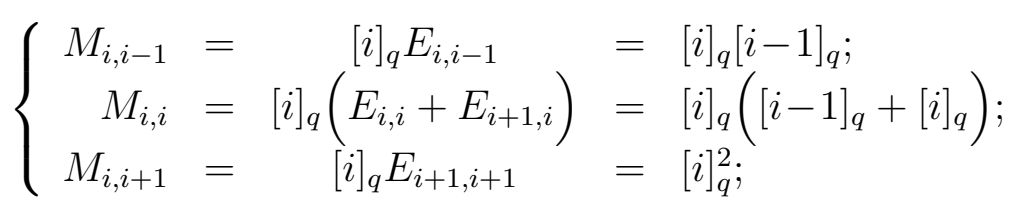

and $M_{i, j}=0$ if $|i-j|>1$. Let $N=E D$. We have

$$
\left\{\begin{aligned}
N_{i, i-1} & =[i-1]_{q}^{2} ; \\
N_{i, i} & =[i-1]_{q}\left([i-1]_{q}+[i]_{q}\right) ; \\
N_{i, i+1} & =[i-1]_{q}[i]_{q} ;
\end{aligned}\right.
$$

and $N_{i, j}=0$ if $|i-j|>1$. For all $i$, we have

$$
\begin{array}{rll}
q N_{i, i-1}+D_{i, i-1}+E_{i, i-1} & =c[i-1]_{q}\left(q[i-1]_{q}+1\right) & =[i-1]_{q}[i]_{q} ; \\
q N_{i, i}+D_{i, i}+E_{i, i} & =\left(q[i-1]_{q}+1\right)\left([i]_{q}+[i-1]_{q}\right) & =[i]_{q}\left([i-1]_{q}+[i]_{q}\right) ; \\
q N_{i, i+1}+D_{i, i+1}+E_{i, i+1} & =\quad\left(q[i-1]_{q}+1\right)[i]_{q} & =[i]_{q}^{2},
\end{array}
$$

which is equal to (16) and so (5) is satisfied.

For (6) and (7), recall that $A$ is described by

$$
\left\{\begin{aligned}
A_{i, i-1} & =q^{i}[i-1]_{q} ; \\
A_{i, i} & =q^{i}\left([i-1]_{q}+[i]_{q}\right) ; \\
A_{i, i+1} & =q^{i}[i]_{q} .
\end{aligned}\right.
$$

Hence, we have

$$
(D A)_{i j}=\sum_{k=1}^{\infty} D_{i k} A_{k j}
$$




$$
=\sum_{k=i}^{i+1}[i]_{q} A_{k j} .
$$

We use the following description:

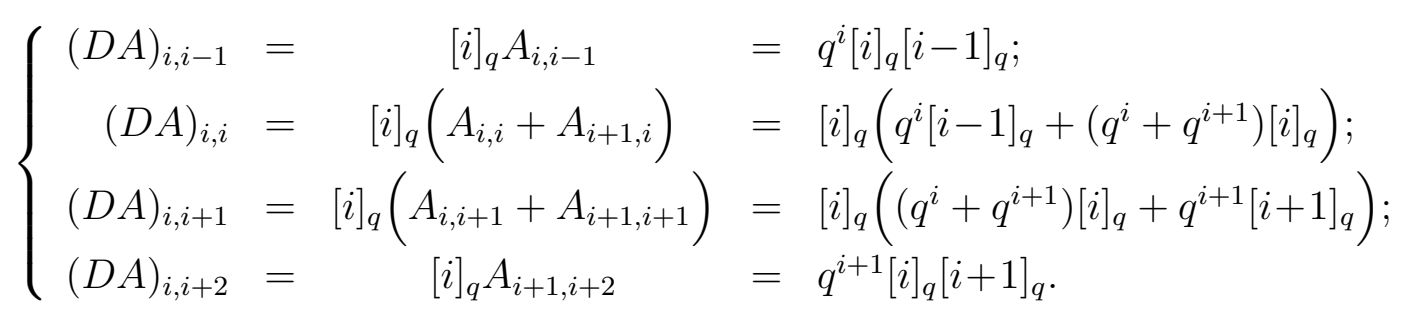

The product $A D$ is described using the same idea:

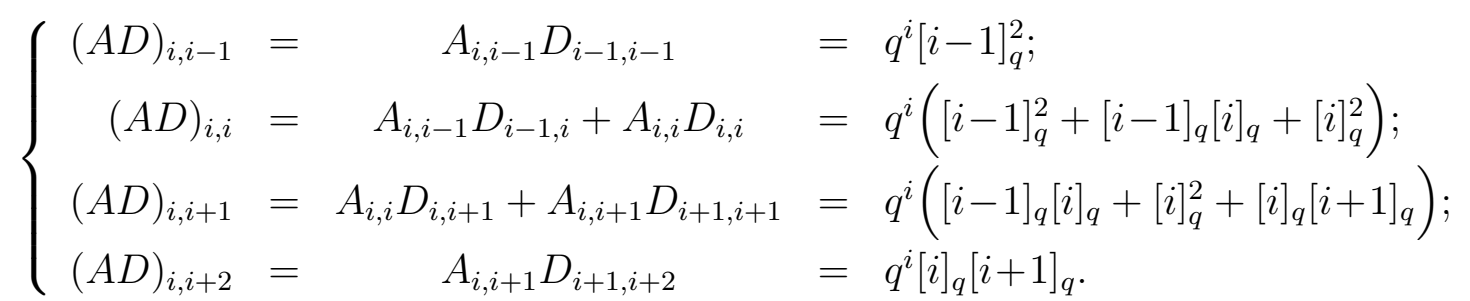

Hence, for all $i$, we have

$$
\begin{aligned}
q(A D)_{i, i-1}+A_{i, i-1} & =q^{i}[i-1]_{q}\left(q[i-1]_{q}+1\right) \\
& =q^{i}[i-1]_{q}[i]_{q} ; \\
q(A D)_{i, i}+A_{i, i} & =q^{i}\left(q[i-1]_{q}+1\right)\left([i]_{q}+[i-1]_{q}\right)+q^{i+1}[i]_{q}^{2} \\
& =q^{i}[i]_{q}\left([i-1]_{q}+[i]_{q}\right)+q^{i+1}[i]_{q}^{2} ; \\
q(A D)_{i, i+1}+A_{i, i+1} & =q^{i}\left(q[i-1]_{q}+1\right)[i]_{q}+q^{i+1}\left([i]_{q}^{2}+[i]_{q}[i+1]_{q}\right) \\
& =q^{i}[i]_{q}^{2}+q^{i+1}\left([i]_{q}^{2}+[i]_{q}[i+1]_{q}\right) \\
q(A D)_{i, i+2}+A_{i, i+2} & =q^{i+1}[i]_{q}[i+1]_{q}+0 \\
& =q^{i+1}[i]_{q}[i+1]_{q},
\end{aligned}
$$

which is equal to (18) and proves that (6) is satisfied.

Let us now consider the product $A E$. We have the following description:

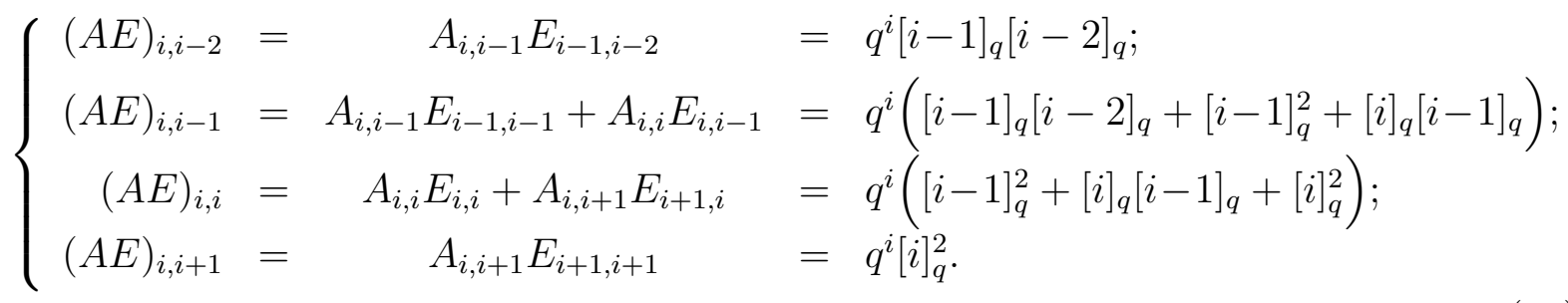


We also have $E A$ described as follow:

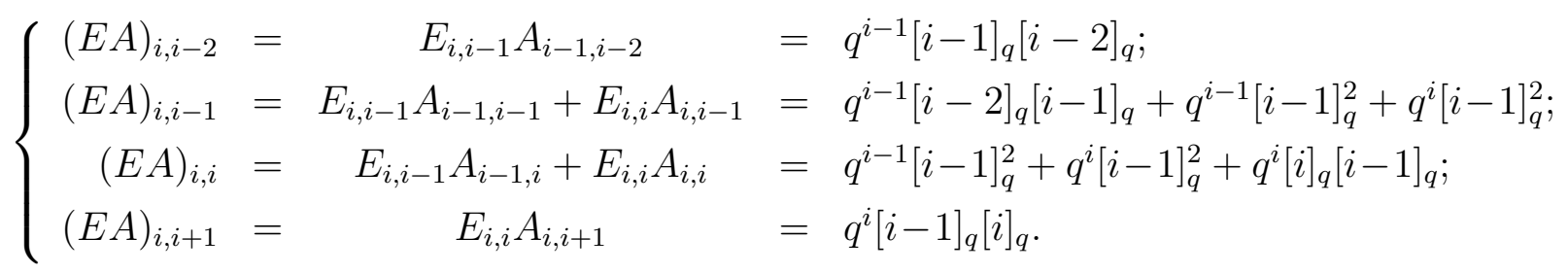

Hence, for all $i$, we have

$$
\begin{aligned}
q(E A)_{i, i-2}+A_{i, i-2} & =q^{i}[i-1]_{q}[i-2]_{q}+0 \\
& =q^{i}[i-1]_{q}[i-2]_{q} \\
q(E A)_{i, i-1}+A_{i, i-1} & =q^{i}[i-1]_{q}[i-2]_{q}+q^{i}[i-1]_{q}^{2}+q^{i}\left(q[i-1]_{q}+1\right)[i-1]_{q} \\
& =q^{i}[i-1]_{q}[i-2]_{q}+q^{i}[i-1]_{q}^{2}+q^{i}[i]_{q}[i-1]_{q} \\
q(E A)_{i, i+1}+A_{i, i+1} & =q^{i}[i-1]_{q}^{2}+q^{i}\left(q[i-1]_{q}+1\right)\left([i-1]_{q}+[i]_{q}\right) \\
& =q^{i}[i-1]_{q}^{2}+q^{i}[i]_{q}\left([i-1]_{q}+[i]_{q}\right) \\
q(E A)_{i, i+2}+A_{i, i+2} & =q^{i}\left(q[i-1]_{q}+1\right)[i]_{q} \\
& =q^{i}[i]_{q}^{2}
\end{aligned}
$$

which is equal to (19) and proves that (7) is satisfied.

Using the structure of the vectors, one easily proves that (8) and (9) are satisfied, which ends the proof.

Remark 14. When $q=1, A=D+E$ satisfies (6) and (7). In this case we can compute the stationary distribution of the 2-PASEP using the stationary distribution of the PASEP (case with zero $\circ$ particles). Let $x$ be a state of the 2-PASEP with $N$ sites and $r$ gray particles. We have

$$
\operatorname{Prob}(x)=\frac{1}{\left(\begin{array}{c}
N \\
r
\end{array}\right)} \sum_{y} \operatorname{Prob}(y),
$$

where the sum is on all the states of the PASEP with particle $\bullet$ at the positions of the particles of $x$, empty sites $\circ$ at the positions of the empty sites $\circ$ of $x$ and a particle $\bullet$ or or an empty site $\circ$ at the sites with a $\bullet$ particle in $x$.

For example, we consider the state $x=\bullet \bullet$. Let $x_{1}=\bullet \bullet \circ$ and $x_{2}=\bullet \circ \circ$ be the states associated. We have $\operatorname{Prob}(x)=\frac{14}{3 \cdot 4 !}$. Similarly, we have $\operatorname{Prob}\left(x_{1}\right)=\operatorname{Prob}\left(x_{2}\right)=\frac{7}{4 !}$, and

$$
\frac{14}{3 \cdot 4 !}=\frac{1}{\left(\begin{array}{l}
3 \\
1
\end{array}\right)}\left(\frac{7}{4 !}+\frac{7}{4 !}\right) .
$$

\section{Path interpretation}

One way to obtain a combinatorial interpretation of the stationary distribution is to interpret each monomial of the numerator and denominator of (10) as a weighted path.

We start by defining a new class of paths generalizing the Laguerre histories. 
Definition 15. A marked Laguerre history of size $(n, r)$ is a Laguerre history of size $n$ where all the steps but the first can be marked and $r$ steps are marked. Any marked step starting from height $h$ increases its weight by $h$.

This way, a step $\longrightarrow$ or $\nearrow$ has a weight between 0 and $h$ if it is not marked and between $h$ and $2 h$ otherwise. A step $\rightarrow$ or $\searrow$ has a weight between 0 and $h-1$ if it is not marked and between $h$ and $2 h-1$ otherwise.

To avoid confusion, all notations for the marked Laguerre histories are represented by bold letters. An example of a marked Laguerre history of size $(8,2)$ is given in Figure 3. The steps with overlined weight are the marked steps.

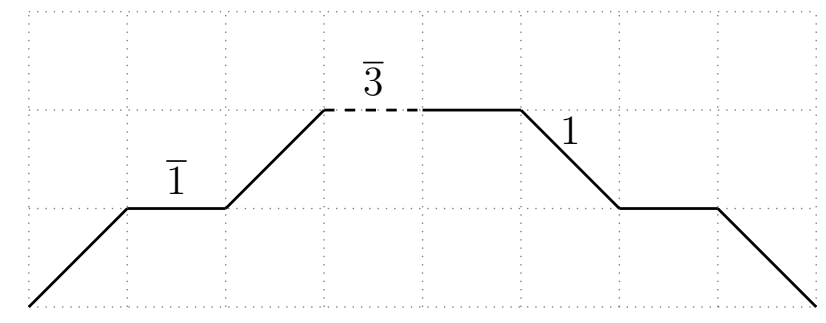

Figure 3: An example of a Laguerre history of $\mathfrak{H}(A D A D E D E)$ with total weight 5.

The total weight of a marked Laguerre history $\mathbf{H}$ (denoted by $\operatorname{tw}(\mathbf{H})$ ) is the sum of the weights of it steps. For example, the total weight of the marked Laguerre history in Figure 3 is 5 . We associate a word of length $n-1$ to a marked Laguerre history $\mathbf{H}$ of length $n$ the following way. The marked steps are labeled by $A$, the $\nearrow$ or $\longrightarrow$ steps are labeled $D$ and the remaining steps are labeled $E$. We forget the label of the first step as it is always $D$. We call this word the label of $\mathbf{H}$ and we denote it by $\operatorname{label}(\mathbf{H})$. For example, the label of the marked Laguerre history in Figure 3 is $A D A D E D E$.

Given a word $X$, let $\mathfrak{H}(X)$ be the set of marked Laguerre histories with label $X$ and let $\mathcal{Z}_{X}$ be the generating polynomial of all the paths:

$$
\mathcal{Z}_{X}(q)=\sum_{H \in \mathfrak{H}(X)} q^{\mathrm{wt}(\mathrm{H})}
$$

and let

$$
\mathcal{Z}_{N, r}(q)=\sum_{X} \mathcal{Z}_{X}(q)
$$

where the sum is over all the words in $\{A, D, E\}^{N}$ with $r$ letters $A$ (with $N=n-1$ ).

The following result gives us a combinatorial interpretation of the steady-state probabilities of the 2-PASEP in terms of marked Laguerre histories.

Theorem 16. Let $x$ be a state of the 2-PASEP with $N$ sites and $r$ gray particles and $X$ be the associated word in $A, D$, and $E$. We have:

$$
\operatorname{Prob}(x)=\frac{\mathcal{Z}_{X}(q)}{\mathcal{Z}_{N, r}(q)}
$$


Proof. The idea is to associate a marked Laguerre history with each monomial of the matrix product of the numerator of (10). Any monomial corresponds to the product of $N$ non-zero coefficients $\left(X_{k}\right)_{i_{k}, j_{k}}$ where $X_{k} \in\{A, D, E\}$ is the matrix corresponding to the $k$ 'th letter of $X$. As the indices $\left(i_{k}, j_{k}\right)$ must satisfy $i_{k}=j_{k-1}$, they can represent the successive heights of a path: $i_{k}$ corresponds to the starting height of the $k$ 'th step and and $j_{k}$ corresponds to its ending height. Moreover, as the matrices $A, D$, and $E$ are tridiagonal, $\left|i_{k}-j_{k}\right| \leqslant 1$ so the paths are Motzkin paths. In order to have a path starting from height 0 , we need to add a $\nearrow$ or $\longrightarrow$ step at the beginning of the path depending on which coefficient of $\langle W|$ has been extracted. For the steps labeled $D$, we have $j_{k} \in\left\{i_{k}, i_{k}+1\right\}$ so the possible steps are $\nearrow$ or $\longrightarrow$; for the steps labeled $E$, we have $j_{k} \in\left\{i_{k}, i_{k}-1\right\}$ so the possible steps are $\searrow$ or $\rightarrow$. For the steps labeled $A$, a coefficient either comes from the matrix $D$ or the matrix $E$. We choose the corresponding step and mark it in order to be able to invert the process.

The weight of the $k$ 'th step of the path corresponds to the power of $q$ taken in the coefficient $\left(X_{k}\right)_{i_{k}, j_{k}}$. One can see that for the matrix $D$ (steps $\nearrow$ or $\longrightarrow$ ) the possible weights are between 0 and $i_{k}$ and that for the matrix $E$ (steps $\searrow$ or $\rightarrow$ ) the possible weights are between 0 and $i_{k}-1$. Finally for the matrix $A$, the weights are the same than for $D$ and $E$ on which we added $i_{k}$ due to the $\operatorname{diag}\left(1, q, q^{2}, q^{3}, \ldots\right)$ factor. This proves that the paths we obtain are exactly the marked Laguerre histories.

Special cases of Theorem 16 are:

$$
\begin{aligned}
\mathcal{Z}_{N, r}(1) & =\left(\begin{array}{c}
N \\
r
\end{array}\right)(N+1) ! \\
\mathcal{Z}_{r, r}(q) & =[r+1]_{q} !
\end{aligned}
$$

The first equation immediately follows from the next section which exhibits a bijection between these marked Laguerre histories and partially signed permutations. The second equation follows from a continued fraction proven by Heine. A bijective proof was given by Biane [2]. Another way to prove it is by using the following lemma.

Lemma 17. If we denote by $m_{n}^{k}(q)$ the sum of the weights of the marked Laguerre histories of size $n$ with $n-1$ marked steps that end at height $k$ (the ending height of the last step is $k$ ), we have

$$
m_{n}^{k}(q)=q^{\left(\begin{array}{c}
k \\
2
\end{array}\right)} \frac{[n]_{q} !^{2}}{[n-k]_{q} ![k]_{q} !}
$$

Proof. We prove this lemma by induction. If $n=1$, the possible values for $k$ are 0 and 1 . In both cases there is only one path of weight 1 as the first step is never marked.

Suppose the property true for $n-1$. A path of length $n$ ending at height $k$ can be either:

- a path of length $n-1$ ending at height $k+1$ followed by a $\searrow$ step;

- a path of length $n-1$ ending at height $k$ followed by a $-\rightarrow$ step or a $\longrightarrow$ step; 
- a path of length $n-1$ ending at height $k-1$ followed by a $\nearrow$ step.

Hence, we have

$$
m_{n}^{k}=m_{n-1}^{k+1} q^{k+1}[k+1]_{q}+m_{n-1}^{k} q^{k}\left([k]_{q}+[k+1]_{q}\right)+m_{n-1}^{k-1} q^{k-1}[k]_{q} .
$$

Using (26) to compute $m_{n-1}^{k+1}, m_{n-1}^{k}$, and $m_{n-1}^{k-1}$ we have the following.

$$
\left\{\begin{aligned}
m_{n-1}^{k+1} & =\frac{[n-1]_{q} !^{2}}{[n-k]_{q} ![k]_{q} !} q^{\left(\begin{array}{c}
k \\
2
\end{array}\right)+2 k+1}[n-k]_{q}[n-k-1]_{q} \\
m_{n-1}^{k} & =\frac{[n-1]_{q} !^{2}}{\left[n-k !_{q}[k]_{q} !\right.} q^{\left(\begin{array}{c}
k \\
2
\end{array}\right)+k}[n-k]_{q}\left([k]_{q}+[k+1]_{q}\right) \\
m_{n-1}^{k-1} & =\frac{[n-1]_{q} !^{2}}{[n-k]_{q} ![k]_{q} !} q^{\left(\begin{array}{c}
k \\
2
\end{array}\right)}[k]_{q}^{2}
\end{aligned}\right.
$$

Using the fact that $q^{k}[n-k]_{q}=[n]_{q}-[k]_{q},(27)$ becomes

$$
q^{\left(\begin{array}{c}
k \\
2
\end{array}\right)} \frac{[n-1]_{q} !^{2}}{[n-k]_{q}[k]_{q}}\left(\left([n]_{q}-[k]_{q}\right)\left([n]_{q}-[k+1]_{q}\right)+\left([n]_{q}-[k]_{q}\right)\left([k]_{q}+[k+1]_{q}\right)+[k]_{q}^{2}\right)
$$

which simplifies to (26).

Note that the special cases $k=0, k=n-1$, and $k=n$ are correctly treated. Indeed, in (28) we have $m_{n-1}^{k+1}$ equals 0 for $k=n$ and $k=n-1$, we have $m_{n-1}^{k}$ equals 0 for $k=n$, and $m_{n-1}^{k-1}$ equals 0 for $k=0$.

We also give a recurrence satisfied by $\mathcal{Z}_{X}(q)$.

Proposition 18. Let $X$ be a word of size $N$ in the letters $A, D$, and $E$ and $s$ be an integer. Denote by $k$ the number of letters $A$ or $E$ in $X$, we have

$$
\begin{aligned}
\mathcal{Z}_{A^{s \cdot D \cdot X}}(q) & =[k+1]_{q} \mathcal{Z}_{A^{s} \cdot X}(q)+\sum_{X=X_{1} \cdot E \cdot X_{2}} q^{\kappa\left(X_{1}\right)} \mathcal{Z}_{A^{s} \cdot X_{1} \cdot D \cdot X_{2}}(q) \\
\mathcal{Z}_{A^{s} \cdot E \cdot X}(q) & =[s+1]_{q} \mathcal{Z}_{A^{s} \cdot X}(q) \\
\mathcal{Z}_{A^{s}}(q) & =[s+1]_{q} !
\end{aligned}
$$

where $A^{s}$ is the word with $s$ times the letter $A$ and $\kappa\left(X_{1}\right)$ is the number of $E$ and $A$ in $X_{1}$.

Proof. Note that $\mathcal{Z}_{A^{s}}(q)=\mathcal{Z}_{r, r}(q)$, such that (31) directly comes from (25).

To prove the other parts of this property, we use the fact that $\mathcal{Z}_{Y}(q)$ is equal to the matrix product $\langle W|m(Y)| V\rangle$ where $m$ is the morphism sending the letters of $Y$ to the matrix satisfying the Ansatz of Proposition 11. We shall identify $m(Y)$ and $Y$ in the rest of this proof.

We prove Equation (30) by induction on $s$. If $s=0$, this equation is just another way of writing (8): $\langle W| E=\langle W|$. Otherwise, using (7), $A E=q E A+A$, we have

$$
\left\langle W\left|A^{s} E X\right| V\right\rangle=q\left\langle W\left|A^{s-1} E A X\right| V\right\rangle+\left\langle W\left|A^{s-1} X\right| V\right\rangle
$$




$$
=\left(q[s]_{q} !+1\right)\left\langle W\left|A^{s} X\right| V\right\rangle,
$$

using the induction relation, so we obtain

$$
\left\langle W\left|A^{s} E X\right| V\right\rangle=[s+1]_{q} !\langle W|S X| V\rangle .
$$

For Equation (29), we prove more generally that for any word $Y$, we have

$$
\langle W|Y D X| V\rangle=[k+1]_{q}\langle W|Y X| V\rangle+\sum_{X=X_{1} E X_{2}} q^{\kappa\left(X_{1}\right)}\left\langle W\left|Y X_{1} D X_{2}\right| V\right\rangle .
$$

It suffices then to set $Y=A^{s}$ to obtain the result. We prove this equation by induction on the size of $X$. If $X$ is the empty word, it is simply another writing of (9): $D|V\rangle=|V\rangle$. Otherwise, let $X^{\prime}$ be the word obtained from $X$ by removing the first letter. There are three possibilities.

- If $X=D X^{\prime}$, let $Y^{\prime}=Y D$. We then have $\langle W|Y D X| V\rangle=\left\langle W\left|Y^{\prime} D X^{\prime}\right| V\right\rangle$. Let us prove that the induction relation applied to $\left\langle W\left|Y^{\prime} D X^{\prime}\right| V\right\rangle$ gives us the same result that for $\langle W|Y D X| V\rangle$. As $X$ and $X^{\prime}$ have the same number of letters $A$ and $E$, the first term of the right part of (32) equals $[k+1]_{q}\left\langle W\left|Y^{\prime} X^{\prime}\right| V\right\rangle$. Moreover, we can write $X$ as $X_{1} E X_{2}$ if and only if we can write $X^{\prime}$ as $X_{1}^{\prime} E X_{2}$ with $X_{1}=D X_{1}^{\prime}$. Moreover, in these equalities, $X_{1}$ and $X_{1}^{\prime}$ have the same number of $E$ and $A$. Thus,

$$
\sum_{X^{\prime}=X_{1}^{\prime} E X_{2}} q^{\kappa\left(X_{1}^{\prime}\right)}\left\langle W\left|Y^{\prime} X_{1}^{\prime} D X_{2}\right| V\right\rangle=\sum_{X=X_{1} E X_{2}} q^{\kappa\left(X_{1}\right)}\left\langle W\left|Y^{\prime} X_{1}^{\prime} D X_{2}\right| V\right\rangle,
$$

so the induction is satisfied using the fact that $Y^{\prime} X^{\prime}=Y X$ and $Y^{\prime} X_{1}^{\prime}=Y X_{1}$.

- If $X=A X^{\prime}$, let $Y^{\prime}=Y A$. Using (6) $(D A=q A D+A)$, we have

$$
\langle W|Y D X| V\rangle=q\left\langle W\left|Y^{\prime} D X^{\prime}\right| V\right\rangle+\langle W|Y X| V\rangle .
$$

As $X^{\prime}$ and $X$ have the same number of letters $E$, we can write $X$ as $X_{1} E X_{2}$ if and only if we can write $X^{\prime}$ as $X_{1}^{\prime} E X_{2}$ with $X_{1}=A X_{1}^{\prime}$. Moreover, in these equalities, $X_{1}$ and $X_{1}^{\prime}$ have the same number of $E$ and $X_{1}$ has one more $A$ than $X_{1}^{\prime}$. Hence, the induction relation implies

$$
\begin{aligned}
\left\langle W\left|Y^{\prime} D X^{\prime}\right| V\right\rangle & =[k]_{q}\left\langle W\left|Y^{\prime} X^{\prime}\right| V\right\rangle+\sum_{X^{\prime}=X_{1}^{\prime} E X_{2}} q^{\kappa\left(X_{1}^{\prime}\right)}\left\langle W\left|Y^{\prime} X_{1}^{\prime} D X_{2}\right| V\right\rangle \\
& =[k]_{q}\langle W|Y X| V\rangle+\sum_{X=X_{1} E X_{2}} q^{\kappa\left(X_{1}\right)-1}\left\langle W\left|Y X_{1} D X_{2}\right| V\right\rangle,
\end{aligned}
$$

as $Y^{\prime} X^{\prime}=Y X$ and $Y^{\prime} X_{1}^{\prime}=Y X_{1}$. Using this equality in (33), the induction relation is satisfied. 
- If $X=E X^{\prime}$, let $Y^{\prime}=Y E$. Using (5) $(D E=q E D+E+D)$, we have

$$
\langle W|Y D X| V\rangle=q\left\langle W\left|Y^{\prime} D X^{\prime}\right| V\right\rangle+\langle W|Y X| V\rangle+\left\langle W\left|Y D X^{\prime}\right| V\right\rangle .
$$

As $X$ starts with an $E$, except for the case $X=E X^{\prime}$, we can write $X$ as $X_{1} E X_{2}$ if and only if $X^{\prime}$ can be written as $X_{1}^{\prime} E X_{2}$ with $X_{1}=A X_{1}^{\prime}$. Moreover, in these equalities, $X_{1}$ and $X_{1}^{\prime}$ have the same number of $A$ and $X_{1}$ has one more $E$ than $X_{1}^{\prime}$. Hence, the induction relation implies:

$$
\left\langle W\left|Y^{\prime} D X^{\prime}\right| V\right\rangle=[k]_{q}\left\langle W\left|Y^{\prime} X^{\prime}\right| V\right\rangle+\sum_{X^{\prime}=X_{1}^{\prime} E X_{2}} q^{\kappa\left(X_{1}^{\prime}\right)}\left\langle W\left|Y^{\prime} X_{1}^{\prime} D X_{2}\right| V\right\rangle,
$$

and the sum can be rewritten as

$$
\sum_{X=X_{1} E X_{2}} q^{\kappa\left(X_{1}\right)-1}\left\langle W\left|Y X_{1} D X_{2}\right| V\right\rangle-\frac{1}{q}\left\langle W\left|Y D X^{\prime}\right| V\right\rangle .
$$

Using this equality in (34), the induction relation is satisfied.

Hence, the induction relation is satisfied in every cases, which ends the proof.

Note that this proof implies that equations (29) and (30) are satisfied for any combinatorial object interpreting the probabilities of the 2-PASEP, whereas Equation (31) depends on the objects themselves.

As a corollary we obtain a factorization of $Z_{X}(q)$.

Corollary 19. Let $X$ be a word of $\{A, D, E\}^{N}$ with $r$ letters $A$. we have

$$
Z_{X}(q)=[r+1]_{q} ! \widetilde{Z_{X}(q)}
$$

where $\widetilde{Z_{X}(q)}$ is a polynomial in $q$ with nonnegative integer coefficients.

It would be interesting to find a combinatorial proof of this result as it would allow us to give a combinatorial interpretation of the probabilities of the 2-PASEP with a general value for $q$ using a smaller family of objects.

In Definition 23 we define an equivalence relation on partially signed permutations implying a combinatorial proof of Corollary 19 for $q=1$.

\section{Combinatorial interpretation using partially signed permuta- tions}

In order to obtain a combinatorial interpretation of these probabilities in terms of generalized permutations, we shall use a generalization of the Françon-Viennot bijection. The original bijection, defined in [14], is a bijection between Laguerre histories and permutations that we extend to a bijection between marked Laguerre histories and partially signed permutations. 
Algorithm 20.

- Input: A partially signed permutation $\boldsymbol{\sigma}$ of size $n$.

- Output: A marked Laguerre history $\mathbf{H}$ of size $n$.

- Execution: Let $H=\psi_{F V}(\sigma)$ where $\sigma$ is the permutation obtained from $\boldsymbol{\sigma}$ by removing the signs. For every $\bar{i} \in \boldsymbol{\sigma}$, mark the $i$ th step of $H$ to build $\mathbf{H}$. When marking a step starting at height $h$, add $h$ to its weight.

We denote by $\Psi_{F V}(\boldsymbol{\sigma})$ the result of this algorithm. For example, for $\boldsymbol{\sigma}=\overline{2} 57836 \overline{4} 1$, the image of $\boldsymbol{\sigma}$ without the signs is given on the left of Figure 4 and the marked version on the right.
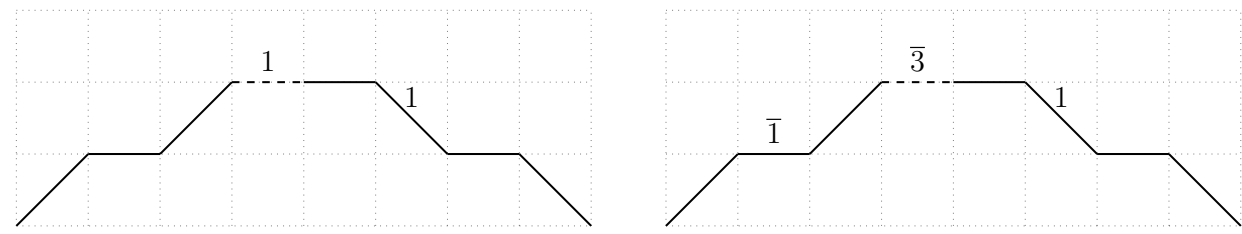

Figure 4: Result of Françon-Viennot bijection for the permutation 25783641 on the left and for the partially signed permutation $\boldsymbol{\sigma}=\overline{2} 57836 \overline{4} 1$ on the right.

The reciprocal map is obtained by storing the positions of the marked steps in the marked Laguerre history and then applying the reciprocal map of the usual FrançonViennot bijection. Finally, just overline the values corresponding to the marked steps.

Proposition 21. The map $\psi_{F V}$ is a bijection between partially signed permutations of size $n$ with $r$ overlined values and marked Laguerre histories of size $n$ with $r$ marked steps. Moreover, let $\boldsymbol{\sigma} \in B_{n}^{\prime}$. We have

$$
\begin{aligned}
\operatorname{ADE}(\operatorname{GC}(\boldsymbol{\sigma})) & =\operatorname{label}\left(\psi_{F V}(\boldsymbol{\sigma})\right) \\
\operatorname{tw}(\boldsymbol{\sigma}) & =\operatorname{tw}\left(\psi_{F V}(\boldsymbol{\sigma})\right) .
\end{aligned}
$$

Proof. As permutations and Laguerre histories are in bijection, there are as many partially signed permutations of size $n$ with $r$ overlined values as marked Laguerre histories of size $n$ with $r$ marked steps. Moreover, let $\boldsymbol{\sigma}$ and $\boldsymbol{\tau}$ be two partially signed permutations having the same image $\mathbf{H}$ by $\psi_{F V}$. As the Françon-Viennot bijection is injective on permutations, the permutations $\sigma$ and $\tau$ obtained from $\boldsymbol{\sigma}$ and $\boldsymbol{\tau}$ are equal. In addition, the positions of the marked steps of $\mathbf{H}$ correspond to the overlined values of $\boldsymbol{\sigma}$ and $\boldsymbol{\tau}$. They are therefore the same and so $\boldsymbol{\sigma}=\boldsymbol{\tau}$.

Let $\boldsymbol{\sigma}$ be a partially signed permutation and $\mathbf{H}=\psi_{F V}(\boldsymbol{\sigma})$, recall from Definition 2 that $\operatorname{tw}(\boldsymbol{\sigma})$ is the number of $31-2$ patterns of $\boldsymbol{\sigma}$ plus its number of $(31, \overline{2})$ patterns. By construction, the number of $31-2$ patterns of $\boldsymbol{\sigma}$ is equal to the weight of $\mathbf{H}$ if we remove 
the additional weights of the marked steps. In order to prove that $\operatorname{tw}(\boldsymbol{\sigma})=\operatorname{tw}(\mathbf{H})$, we prove that the height $h$ of a marked step of $\mathbf{H}$ in position $k$ is equal to the number of descents $\boldsymbol{\sigma}_{i}>\boldsymbol{\sigma}_{i+1}$ of $\boldsymbol{\sigma}$ such that $\boldsymbol{\sigma}_{i+1}<k<\boldsymbol{\sigma}_{i}$. We use the reciprocal map of the Françon-Viennot bijection recalled in Algorithm 7 where $h$ corresponds to the number of $\circ$ present when we add the value $k$ in the permutation. Moreover, all these positions except for the last one shall be occupied by a value greater than $k$ and shall create a descent with the value to its right. Thus, there are $h$ descents satisfying the desired property.

Finally, depending if $k$ is overlined or not and forms a descent or not, the $k$ 'th step of $H$ is labeled by $A, E$, or $D$ and $\operatorname{ADE}(\operatorname{GC}(\boldsymbol{\sigma}))$ is equal to the label of $\psi_{F V}(\boldsymbol{\sigma})$.

Using Theorem 16 and Proposition 21 we obtain the following theorem.

Theorem 22. Let $x$ be a state of the 2-PASEP with $N$ sites having $r$ gray particles. We have

$$
\operatorname{Prob}(x)=\frac{1}{Z_{N, r}(q)} \sum_{\operatorname{ADE}(\operatorname{GC}(\boldsymbol{\sigma}))=X(x)} q^{\mathrm{tw}(\boldsymbol{\sigma})},
$$

where $Z_{N, r}$ is the sum of $q^{\mathrm{tw}(\boldsymbol{\sigma})}$ for all partially signed permutations $\boldsymbol{\sigma}$ of size $N+1$ having $r$ overlined values

Hence, partially signed permutations can be used to describe the probabilities of the 2-PASEP for any value of the parameter $q$.

In order to give a combinatorial proof of Corollary 19 in the case $q=1$, we define here an equivalence relation on partially signed permutations.

Definition 23. Let $\boldsymbol{\sigma}$ be a partially signed permutation of size $n$ with $r$ overlined values. Let $i_{1}, \ldots, i_{r+1}$ be the positions of these values and of 1 , and let $u_{1}, \ldots, u_{r+2}$ be the factors of $\boldsymbol{\sigma}$ of the form

$$
u_{k}:=\boldsymbol{\sigma}_{i_{k-1}+1} \ldots \boldsymbol{\sigma}_{i_{k}},
$$

with $i_{0}=0$ and $i_{r+2}=n$. Let $\boldsymbol{\tau}$ be a partially signed permutation, we say that $\boldsymbol{\sigma}$ and $\boldsymbol{\tau}$ are equivalent $(\boldsymbol{\sigma} \sim \boldsymbol{\tau})$ if there is a permutation $\mu \in \mathfrak{S}_{r+1}$ such that

$$
\tau=u_{\mu_{1}} \ldots u_{\mu_{r+1}} u_{r+2} .
$$

For example, for $\boldsymbol{\sigma}=\overline{2} 73 \overline{4} 5186$, we have $u_{1}=\overline{2}, u_{2}=73 \overline{4}, u_{3}=51$ and $u_{4}=86$. For $\mu=312$, we have $\boldsymbol{\tau}=51 \overline{2} 73 \overline{4} 86$. The set of the partially signed permutations equivalent to $\sigma$ is:

$\{\overline{2} 5173 \overline{4} 86, \overline{2} 73 \overline{4} 5186,51 \overline{2} 73 \overline{4} 86,5173 \overline{42} 86,73 \overline{42} 5186,73 \overline{4} 51 \overline{2} 86\}$.

Lemma 24. Let $\boldsymbol{\sigma}$ and $\boldsymbol{\tau}$ be two partially signed permutations such that $\boldsymbol{\sigma} \sim \boldsymbol{\tau}$. We have

$$
\mathrm{GC}(\boldsymbol{\sigma})=\mathrm{GC}(\boldsymbol{\tau})
$$


Proof. Firstly, we have $\operatorname{Sign}(\boldsymbol{\sigma})=\operatorname{Sign}(\boldsymbol{\tau})$ as $\sim$ does not change the signs of the values. Moreover, the value on the right of a not overlined value (other than 1 ) is the same in $\boldsymbol{\sigma}$ and in $\boldsymbol{\tau}$ such that $\operatorname{GDes}(\boldsymbol{\sigma})=\operatorname{GDes}(\boldsymbol{\tau})$, which ends the proof.

As there are $(r+1)$ ! partially signed permutations in the equivalence class of a permutation with $r$ overlined values, this lemma implies Corollary 19 for $q=1$. Note that the above equivalence relation leaves GC unchanged but does not modify the weight in order to obtain the $[r+1]_{q}$ ! factor.

Each equivalence class may be identified by an assemblée of permutation, a family of object used in [18] to give a combinatorial interpretation of the probabilities of the 2-PASEP in the case $q=1$ with other parameters.

\section{Another interpretation}

In Section 3 we show that for any state $x$ of the 2-PASEP and $X=X(x)$, we have $\operatorname{Prob}(x)=\operatorname{Prob}(\iota(x))$ where $\iota$ is the particle hole symmetry involution of Definition 10 .

Unfortunately, this property cannot be observed directly on the marked Laguerre histories and on the partially signed permutations. In the case of the usual PASEP, this property also exists and can be observed using large Laguerre histories [16]. Using the connection between Laguerre histories and large Laguerre histories, we define the marked large Laguerre histories and use them to obtain another interpretation of the probabilities.

\subsection{Marked large Laguerre histories}

Let us start by recalling the definition of large Laguerre histories [29].

Definition 25. A large Laguerre history of size $n$ is a weighted Motzkin path of size $n$ with two different horizontal steps such that the weight of a step $\nearrow, \longrightarrow$, \, or $-\rightarrow$ starting from height $h$ is between 0 and $h$.

Definition 26. A marked large Laguerre history of size $(n, r)$ is a large Laguerre history of size $n$ such that every step may be marked and $r$ steps are marked. If a step $\nearrow$ or $\longrightarrow$ starting at height $h$ is marked, its weight is increased by $h+1$ where the marked steps $\searrow$ or $\rightarrow$ starting at height $h$ increase their weight by $h$.

Hence, a step $\longrightarrow$ or $\nearrow$ has a weight between 0 and $h$ if it is not marked and between $h+1$ and $2 h+1$ if it is marked. A step $\rightarrow$ or $\searrow$ has a weight between 0 and $h$ if it is not marked and between $h$ and $2 h$ if it is marked.

The label of a marked large Laguerre history $\mathbf{H}$ of size $(n, r)$ is the word of size $n$ in the letters $A, D$ and $E$ obtained by sending the marked steps on $A$, the steps $\nearrow$ and $\longrightarrow$ on $D$ and the remaining steps on $E$. We denote it by label $(\mathbf{H})$.

Note that the marked large Laguerre histories of size $(n, 0)$ are exactly the large Laguerre histories of size $n$.

An example of a marked large Laguerre history is given in Figure 5. We denote by $\mathfrak{H}^{0}(X)$ the set of all marked large Laguerre histories whose label is $X$. 


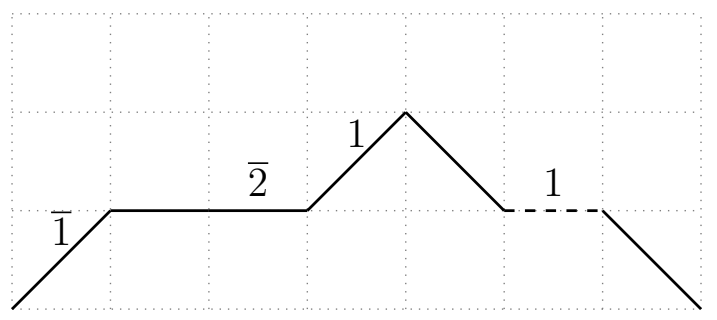

Figure 5: A marked large Laguerre history of size $(8,2)$ which label is $A D A D D E D E$.

In order to use these objects to obtain a combinatorial interpretation of the probabilities of the 2-PASEP, we define a bijection between marked Laguerre histories and marked large Laguerre histories. This map is a generalization of a bijection between Laguerre histories and large Laguerre histories described by the second author in [22] using weighted Dyck path. The following algorithm is another description of this map directly on Laguerre histories.

Algorithm 27.

- Input: a Laguerre history $H$ of size $n$.

- Output: a large Laguerre history $H^{\prime}$ of size $n-1$.

- Execution: for all $1 \leqslant i<n$, build $H_{i}^{\prime}$ (the $i$ th step of $H^{\prime}$ ) using the following table

\begin{tabular}{c|c|c}
$H_{i} \backslash H_{i+1}$ & $\nearrow$ or $\longrightarrow$ & $\searrow$ or $\rightarrow$ \\
\hline$\nearrow$ or $\rightarrow$ & $\nearrow$ & $-\rightarrow$ \\
\hline$\searrow$ or $\longrightarrow$ & $\longrightarrow$ & $\searrow$
\end{tabular}

The weight of $H_{i}^{\prime}$ is equal to the weight of $H_{i}$.

We denote by $\Psi$ the map associated with Algorithm 27. The reciprocal map can also be described using a similar table from large Laguerre histories of size $n$ to Laguerre histories of size $n+1$. An example of $\Psi$ is given in Figure 6

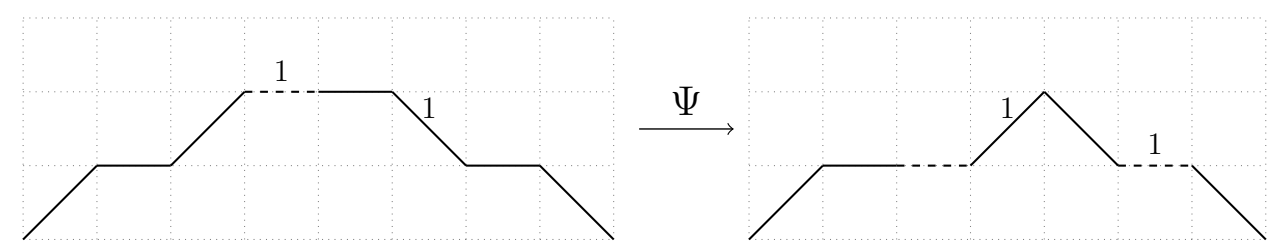

Figure 6: An example of $\Psi$ on a Laguerre history of size 8. 
The map $\Psi$ is a variant of a well known bijection between Laguerre histories and large Laguerre histories using their common representation as weighted Dyck paths [22]. The proof that it is a bijection uses the following lemma.

Lemma 28. Let $H$ be a Laguerre history of size $n$ and $0<k<n$. Let $h_{k}$ be the height of the k'th step of $H$ and $h_{k}^{\prime}$ be the height of the $k$ 'th step of $\Psi(H)$

- If $H_{k}$ is $\nearrow$ or $\longrightarrow$, then $h_{k}^{\prime}=h_{k}$.

- If $H_{k}$ is $\searrow$ or $\rightarrow$, then $h_{k}^{\prime}=h_{k}-1$.

Proof. We prove this lemma by induction on $k$. Let $H^{\prime}=\Psi(H)$. We have $h_{1}=h_{1}^{\prime}=0$ and $H_{1}$ is indeed $\nearrow$ or $\longrightarrow$. In the case $k=2$, if $H_{2}$ is $\nearrow$ or $\longrightarrow$ then, using the table in (42), $H_{1}^{\prime}=H_{1}$ as it cannot be $\searrow$ or $\rightarrow \rightarrow$. This proves that $h_{2}=h_{2}^{\prime}$. Moreover, if $H_{2}$ is $\searrow$ or $\rightarrow$ then $H_{1}$ is necessarily $\nearrow$ and so $H_{1}^{\prime}$ is $\rightarrow$ and $h_{2}^{\prime}=0=h_{2}-1$.

Suppose the lemma true for $k-1$, there are four cases obtained directly from (42).

- If $H_{k-1}$ is $\nearrow$ or $\longrightarrow$ and $H_{k}$ is $\nearrow$ or $\longrightarrow$ then $H_{k-1}^{\prime}=H_{k-1}$ and $h_{k-1}^{\prime}=h_{k-1}$ which implies $h_{k}^{\prime}=h_{k}$.

- If $H_{k-1}$ is $\searrow$ or $-\rightarrow$ and $H_{k}$ is $\nearrow$ or $\longrightarrow$ then $H_{k-1}^{\prime}=H_{k-1}$ and $h_{k-1}^{\prime}=h_{k-1}-1$ which implies $h_{k}^{\prime}=h_{k}-1$.

- If $H_{k-1}$ is $\nearrow$ or $\longrightarrow$ and $H_{k}$ is $\searrow$ or $\rightarrow$ then $h_{k-1}^{\prime}=h_{k-1}$ and $h_{k}^{\prime}=h_{k}-1$.

- If $H_{k-1}$ is $\searrow$ or $\rightarrow$ and $H_{k}$ is $\searrow$ or $\rightarrow$ then $h_{k-1}^{\prime}=h_{k-1}-1$ and $h_{k}^{\prime}=h_{k}$.

Lemma 29. Let $H$ be a Laguerre history. We have

$$
\begin{aligned}
\operatorname{label}(H) & =\operatorname{label}(\Psi(H)) ; \\
\operatorname{tw}(H) & =\operatorname{tw}(\Psi(H)) .
\end{aligned}
$$

Proof. The weight stays unchanged when applying $\Psi$ which proves Equation (44).

Moreover, using (42) we have that for $i<n$, the $i$ 'th step of $\Psi(H)$ is $\longrightarrow$ or $\nearrow$ if and only if it is the case of the $(i+1)$-st step of $H$. This proves (43).

Note that this result is a reformulation of the first two points of Proposition 3.6 of [22].

Let us now extend this map to the marked versions of these objects.

Algorithm 30.

- Input: a marked Laguerre history $\mathbf{H}$.

- Output: a marked large Laguerre history $\mathbf{H}^{\prime}$.

- Execution:

- let $H$ be the Laguerre history obtained from $\mathbf{H}$ by removing its marks;

- $H^{\prime}=\Psi(H)$; 
- for all $k$ such that $\mathbf{H}_{k}$ is marked, mark the $(k-1)^{\prime}$ th step of $H^{\prime}$ to build $\mathbf{H}^{\prime}$;

- for all marked steps of $\mathbf{H}$, if it is a $\longrightarrow$ (resp. $-\rightarrow$ ), change it in $\rightarrow$ (resp. $\longrightarrow)$.

As the execution of this algorithm is the same as the execution of Algorithm 27 when applied to Laguerre history, this one is a generalization to marked Laguerre histories that we also denote by $\Psi$. An example of an execution of $\Psi$ on a marked Laguerre history is given on Figure 7. In this example, the Laguerre history $H$ and the large Laguerre history $H^{\prime}$ are the ones of Figure 6.

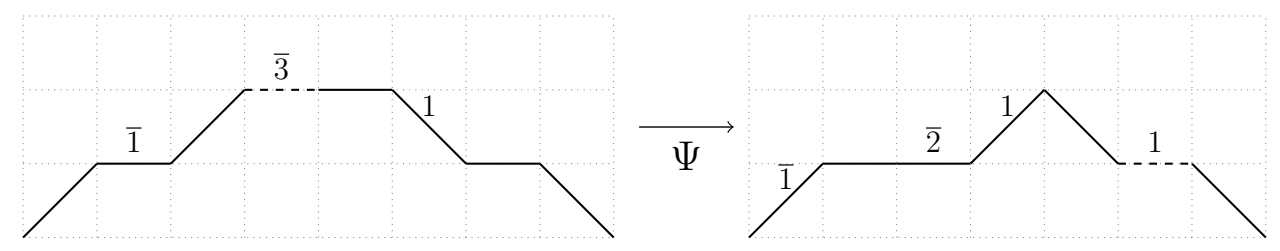

Figure 7: A marked Laguerre history on the left and its image by $\Psi$ on the right. The intermediate steps correspond to the paths in Figure 6.

Proposition 31. The map $\Psi$ sending marked Laguerre histories to marked large Laguerre histories is a bijection. Moreover, for every marked Laguerre history $\mathbf{H}$, we have

$$
\begin{aligned}
\operatorname{label}(\mathbf{H}) & =\operatorname{label}(\Psi(\mathbf{H})) \\
\operatorname{tw}(\mathbf{H}) & =\operatorname{tw}(\Psi(\mathbf{H})) .
\end{aligned}
$$

Proof. The fact that $\Psi$ is a bijection comes directly from the fact that $\Psi$ is a bijection from Laguerre histories to large Laguerre histories.

As the positions of the letters $A$ are the same in label $(\mathbf{H})$ and in $\operatorname{label}(\Psi(\mathbf{H}))$ and that the non-marked steps of $\psi(\mathbf{H})$ are the same as the steps of $\psi(H)$, Equation (45) is a consequence of Lemma 29.

Let $\mathbf{H}^{\prime}=\Psi(\mathbf{H})$. Equation (44) implies that we only need to prove that the weight we add to a marked step of $\mathbf{H}^{\prime}$ corresponds to the added weight as we mark the corresponding step in $\mathbf{H}$ in order to prove (46).

Let $\left(h_{1}, \ldots, h_{n}\right)$ and $\left(h_{1}^{\prime}, \ldots, h_{n-1}^{\prime}\right)$ be such that $h_{i}$ (resp. $\left.h_{i}^{\prime}\right)$ is the height of the $i$ 'th step of $\mathbf{H}$ (resp. $\left.\mathbf{H}^{\prime}\right)$. Let $k$ be the position of a marked step of $\mathbf{H}$. Let $H$ be the Laguerre history obtained from $\mathbf{H}$ by removing the marked steps and their additional weight and $H^{\prime}$ the one obtained from $\mathbf{H}^{\prime}$. As we exchange the steps $\longrightarrow$ and $\rightarrow$ when we mark a step and as the $\longrightarrow$ and $\nearrow$ have a greater weight increase when marked on large Laguerre histories, we need to prove that $h_{k-1}^{\prime}=h_{k}-1$ if $H_{k-1}^{\prime}$ is a $\nearrow$ step or a $\rightarrow-\rightarrow$ step and that $h_{k-1}^{\prime}=h_{k}$ otherwise. Let us treat the four different cases using Lemma 28 and (42).

- If $H_{k-1}^{\prime}$ is $\nearrow$ we have $h_{k-1}^{\prime}=h_{k}^{\prime}-1$. Moreover, $H_{k}$ is $\nearrow$ step or $\longrightarrow$ so $h_{k}^{\prime}=h_{k}$ and then $h_{k-1}^{\prime}=h_{k}-1$. 
- If $H_{k-1}^{\prime}$ is $\rightarrow$ we have $h_{k-1}^{\prime}=h_{k}^{\prime}$. Moreover, $H_{k}$ is $\searrow$ or $\rightarrow$ so $h_{k}^{\prime}=h_{k}-1$ and then $h_{k-1}^{\prime}=h_{k}-1$.

- If $H_{k-1}^{\prime}$ is $\longrightarrow$ we have $h_{k-1}^{\prime}=h_{k}^{\prime}$. Moreover, $H_{k}$ is $\nearrow$ step or $\longrightarrow$ so $h_{k}^{\prime}=h_{k}$ and then $h_{k-1}^{\prime}=h_{k}$.

- If $H_{k-1}^{\prime}$ is $\searrow$ we have $h_{k-1}^{\prime}=h_{k}^{\prime}+1$. Moreover, $H_{k}$ is $\searrow$ or $\rightarrow$ so $h_{k}^{\prime}=h_{k}-1$ and then $h_{k-1}^{\prime}=h_{k}$.

We then deduce the following combinatorial interpretation.

Corollary 32. Let $x$ be a state of the 2-PASEP with $N$ sites and $r$ gray particles. We have

$$
\operatorname{Prob}(x)=\frac{1}{Z_{N, r}(q)} \sum_{\mathbf{H} \in \mathfrak{H}^{0}(X(x))} q^{\mathrm{tw}(\mathbf{H})},
$$

where $Z_{N, r}(q)$ is the generating series of the weights of the marked large Laguerre histories of size $N$ with $r$ marked steps.

\subsection{An involution on Laguerre histories}

Our main interest in this section is to describe an involution on marked (large) Laguerre histories that behaves the same way as $\iota$ does on the states of the 2-PASEP where $\iota$ is the map defined in Definition 10. This goal is easier to achieve on marked large Laguerre histories.

To describe this involution we need the notion of opposing steps of a path: given $H_{i}=\nearrow$, the opposing step is the $H_{j}=\searrow$ such that

$$
j=\min _{k>i}\left\{H_{k}=\searrow \mid h_{k}=h_{i}+1\right\} .
$$

We represented all opposing steps of a path in Figure 8. For example, the opposing step of $H_{5}$ is $H_{9}$.

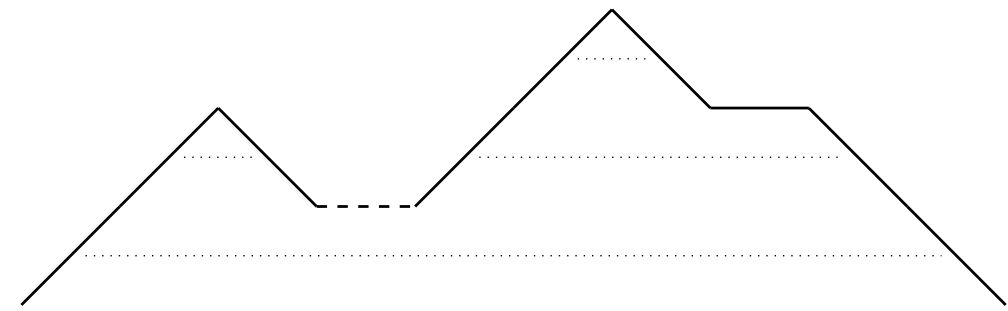

Figure 8: All opposing steps of a path.

Algorithm 33.

- Input: a marked large Laguerre history $\mathbf{H}$.

- Output: a marked large Laguerre history $\widetilde{\mathbf{H}}$. 
- Execution: Let $i_{1}, \ldots, i_{r}$ be the positions of the marked steps of $\mathbf{H}$. Apply the following steps:

1. unmark the path;

2. reverse the path $H$;

3. exchange the weights of the opposing $\nearrow$ and $\searrow$;

4. mark the path at the positions $n-i_{1}, \ldots, n-i_{r}$;

5. change the unmarked $\longrightarrow$ to $\rightarrow-\rightarrow$ and reciprocally.

We denote by $\iota$ the map associated with Algorithm 33. A detailed example of this transformation is given in Figure 9 with the different intermediate steps representing which step is applied between two paths.

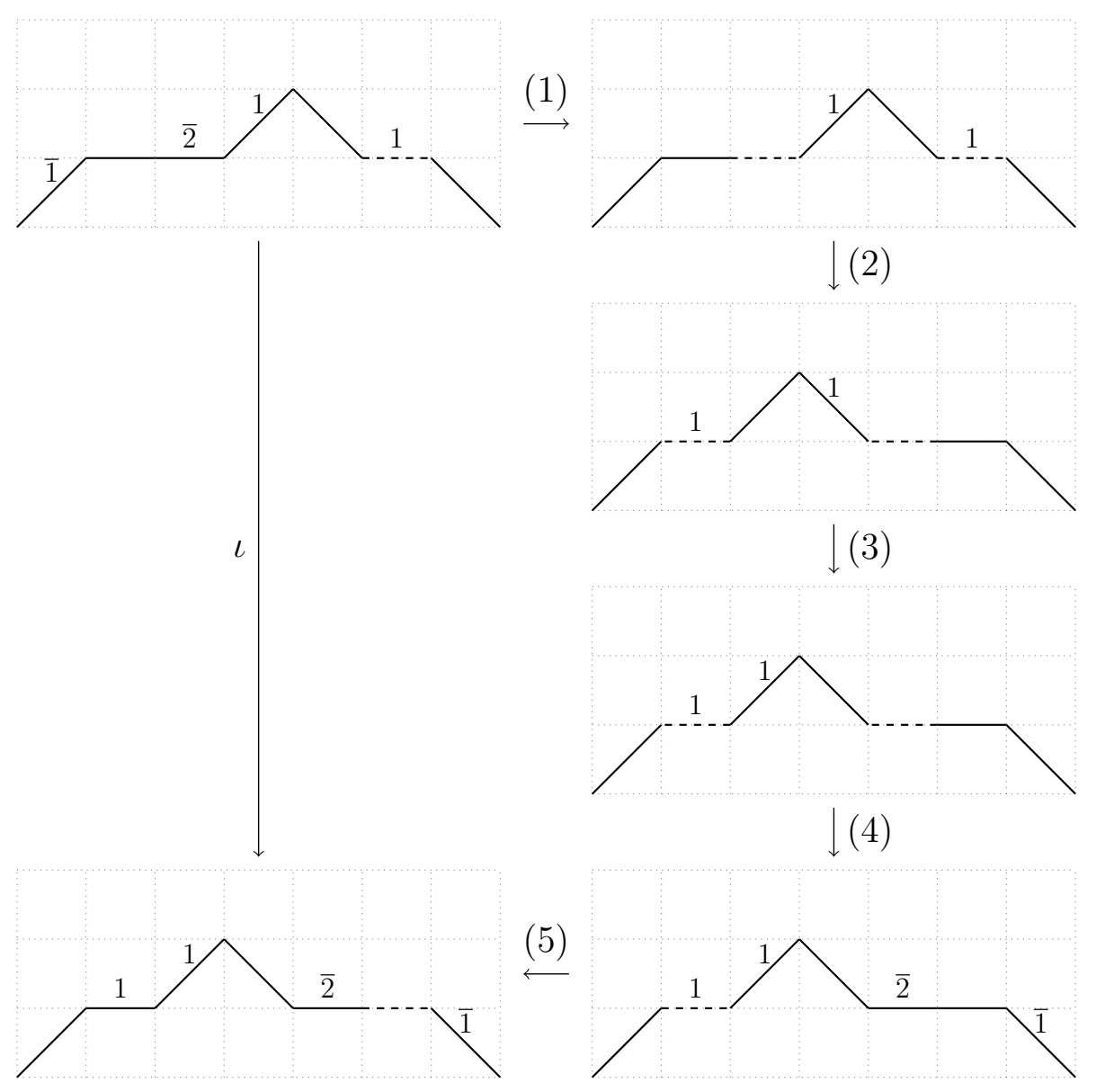

Figure 9: An execution of $\iota$ on $\mathbf{H} \in \mathfrak{H}^{0}(A D A D E E E)$ to its image $\widetilde{\mathbf{H}} \in \mathfrak{H}^{0}(D D D E A E A)$. 
Theorem 34. The map $\iota$ is an involution on marked large Laguerre histories. Moreover, if $\mathbf{H}$ is a marked large Laguerre history, we have

$$
\begin{aligned}
\iota(\operatorname{label}(\mathbf{H})) & =\operatorname{label}(\iota(\mathbf{H})) ; \\
\operatorname{tw}(\mathbf{H}) & =\operatorname{tw}(\iota(\mathbf{H})) .
\end{aligned}
$$

Proof. Reversing a Laguerre history exchanges the starting height of a step with its ending height. For horizontal steps this doesn't change anything. For increasing and decreasing steps, exchanging the weight with the opposing step ensure that the weight of every step is between 0 and its starting height after reversing the path. Hence, $\iota$ is an involution.

Reversing the path changes a $\searrow$ step to a $\nearrow$ one and conversely. Exchanging $\longrightarrow$ by $\rightarrow \rightarrow$ and conversely corresponds to exchanging $D$ and $E$ in the definition of $\iota$ on words in $\{A, D, E\}$. Moreover, the positions of the letter $A$ is just reversed which proves (48).

The steps one, two, and four of Algorithm 33 do not change the weight of the path. We only need to prove that marking $H$ at a position $n-i_{k}$ increases the weight as much as it is decreased by unmarking $\mathbf{H}$ at position $i_{k}$. If the step is $\longrightarrow$ or $-\rightarrow$, its starting height does not change through the different steps. The steps does not change either. The increased weight is the same. A step $\nearrow$ is sent to $\searrow$ by reversing the path and conversely, this increases or decreases the height by one which compensate the fact that, starting at height $h$ a step $\nearrow$ increases its weight by $h+1$ and a step $\searrow$ by $h$. This proves (49).

The maps $\Psi$ and $\iota$ also induce an involution directly on marked Laguerre history.

Corollary 35. Let $\mathbf{H}$ be a marked Laguerre history and $\mathrm{i}=\Psi^{-1} \circ \iota \circ \Psi$, we have

$$
\begin{aligned}
\iota(\operatorname{label}(\mathbf{H})) & =\operatorname{label}(\mathrm{i}(\mathbf{H})) \\
\operatorname{tw}(\mathbf{H}) & =\operatorname{tw}(\mathrm{i}(\mathbf{H})) .
\end{aligned}
$$

\section{Conclusion}

This combinatorial work gives rise to an algebraic interpretation developed by the second author in [23]. Thanks to this combinatorial algebra setting Nunge gives an exact enumeration formula of the stationary distribution of any state of the 2-ASEP. This generalizes results on noncommutative symmetric functions [15, 26, 21].

Theorem 36. [23] Let $x$ be a state of the 2-PASEP and $\mathbf{I}$ be the segmented composition such that $X(x)=\operatorname{ADE}(\mathbf{I})$. We have

$$
Z_{X(x)}=\sum_{\mathbf{J} \preceq \mathbf{I}}\left(\frac{-1}{q}\right)^{\ell(\mathbf{I})-\ell(\mathbf{J})} q^{-\mathrm{st}^{\prime}(\mathbf{I}, \mathbf{J})} c_{\mathbf{J}},
$$

where $c_{\mathbf{J}}=[s]_{q}^{j_{1}}[s-1]_{q}^{j_{2}} \ldots[1]_{q}^{j_{s}}$ with $j_{1}, \ldots, j_{s}$ are the parts of $\mathbf{J}$ and $\mathrm{st}^{\prime}$ is a statistic on segmented compositions. 
The 2-PASEP has five parameters in its most general form [27] $\alpha, \beta, \gamma, \delta$ and $q$. In this work we set $\alpha=\beta=1$ and $\gamma=\delta=0$. We could also set the probabilities equal to $1 /(N+1)$ to be equal to $u /(N+1)$ and then choose for example set $q>1$ and adjust the other parameters. This is an interesting problem suggested by the referee. Some of our results could probably be generalized in the case for $\alpha$ and $\beta$ general. The work of Josuat-Vergès in the case of the classical PASEP [16] gives an interpretation for the Laguerre histories and the permutations. We leave as an open problem to generalize this to marked Laguerre histories and partially signed permutations.

Inspired by the Markov chains defined on permutations (or permutation tableaux) that project on the PASEP [7], we would like to define an analog on the partially signed permutations or on marked Laguerre histories. We conjecture that it is possible to define such a Markov chain on partially signed permutations with $r$ signs that will project to the 2-PASEP. Ideally the graph would be composed of $(r+1)$ ! components. Each component would project to the Markov chain of 2-PASEP.

A natural generalization of the 2-PASEP is due to Cantini [4]. Given $\ell$ a positive integer, the state of the process is all the words of length $N$ on the alphabet $\{-\ell, \ldots, \ell\}$. Then particles $i j$ can become $j i$ with rate 1 if $i>j$ and with rate $q$ otherwise. At the left and right border a particle $i$ can become $-i$ with a certain rate. The 2-PASEP is the case $\ell=1$. The matrix ansatz does not hold in this general setting but Cantini shows that the partition function is a specialization of a Koornwinder polynomial [4]. We leave as an open problem to generalize partially signed permutations to this model.

\section{Acknowledgements}

The authors would like to thank Matthieu Josuat-Vergès and Lenny Tevlin for helpful discussions during the elaboration of this work. They would also thank the anonymous referee for their constructive comments. Finally they thank IRIF, CNRS and Université de Paris where this work was elaborated.

\section{References}

[1] E. Babson and E. Steingrímsson. Generalized permutation patterns and a classification of the Mahonian statistics. Sém. Lothar. Combin., 44:Art. B44b, 18, 2000.

[2] P. Biane. Permutations suivant le type d'excédance et le nombre d'inversions et interprétation combinatoire d'une fraction continue de Heine. European J. Combin., 14(4):277-284, 1993.

[3] Peter Buchholz. Exact and ordinary lumpability in finite Markov chains. J. Appl. Probab., 31(1):59-75, 1994.

[4] L. Cantini. Asymmetric simple exclusion process with open boundaries and Koornwinder polynomials. Ann. Henri Poincaré, 18(4):1121-1151, 2017.

[5] S. Corteel. Crossings and alignments of permutations. Adv. in Appl. Math., 38(2):149-163, 2007. 
[6] S. Corteel, O. Mandelshtam, and L. K. Williams. Combinatorics of the two-species ASEP and Koornwinder moments. Adv. Math., 321:160-204, 2017.

[7] S. Corteel and L. K. Williams. A Markov chain on permutations which projects to the PASEP. Int. Math. Res. Not. IMRN, (17):Art. ID rnm055, 27, 2007.

[8] S. Corteel and L. K. Williams. Tableaux combinatorics for the asymmetric exclusion process. Adv. in Appl. Math., 39(3):293-310, 2007.

[9] S. Corteel and L. K. Williams. Tableaux combinatorics for the asymmetric exclusion process and Askey-Wilson polynomials. Duke Math. J., 159(3):385-415, 2011.

[10] S. Corteel and L. K. Williams. Macdonald-Koornwinder moments and the two-species exclusion process. Selecta Math. (N.S.), 24(3):2275-2317, 2018.

[11] A. de Médicis and X. G. Viennot. Moments des q-polynômes de Laguerre et la bijection de Foata-Zeilberger. Adv. in Appl. Math., 15(3):262-304, 1994.

[12] B. Derrida, M. R. Evans, V. Hakim, and V. Pasquier. Exact solution of a 1D asymmetric exclusion model using a matrix formulation. J. Phys. A, 26(7):1493-1517, 1993.

[13] D. Foata and D. Zeilberger. Denert's permutation statistic is indeed Euler-Mahonian. Stud. Appl. Math., 83(1):31-59, 1990.

[14] J. Françon and G. Viennot. Permutations selon leurs pics, creux, doubles montées et double descentes, nombres d'Euler et nombres de Genocchi. Discrete Math., 28(1):2135, 1979.

[15] F. Hivert, J.-C. Novelli, L. Tevlin, and J.-Y. Thibon. Permutation statistics related to a class of noncommutative symmetric functions and generalizations of the Genocchi numbers. Selecta Math. (N.S.), 15(1):105-119, 2009.

[16] M. Josuat-Vergès. Combinatorics of the three-parameter PASEP partition function. Electron. J. Combin., 18(1):\#P22, 2011.

[17] John G. Kemeny and J. Laurie Snell. Finite Markov chains. Springer-Verlag, New York-Heidelberg, 1976. Reprinting of the 1960 original, Undergraduate Texts in Mathematics.

[18] O. Mandelshtam and X. Viennot. Rhombic alternative tableaux and assemblées of permutations. European J. Combin., 73:1-19, 2018.

[19] O. Mandelshtam and X. Viennot. Tableaux combinatorics of the two-species PASEP. J. Combin. Theory Ser. A, 159:215-239, 2018.

[20] P. Nadeau. The structure of alternative tableaux. J. Combin. Theory Ser. A, 118(5):1638-1660, 2011.

[21] J.-C. Novelli, J.-Y. Thibon, and L. K. Williams. Combinatorial Hopf algebras, noncommutative Hall-Littlewood functions, and permutation tableaux. Adv. Math., 224(4):1311-1348, 2010.

[22] A. Nunge. An equivalence of multistatistics on permutations. J. Combin. Theory Ser. A, 157:435-460, 2018. 
[23] A. Nunge. Monomial basis for hopf algebra on segmented compositions and $q$-analog. In preparation, 2019.

[24] R. Simion and D. Stanton. Octabasic Laguerre polynomials and permutation statistics. J. Comput. Appl. Math., 68(1-2):297-329, 1996.

[25] E. Steingrímsson and L. K. Williams. Permutation tableaux and permutation patterns. J. Combin. Theory Ser. A, 114(2):211-234, 2007.

[26] L. Tevlin. Noncommutative analogs of monomial symmetric functions, cauchy identity, and hall scalar product. Proceedings of FPSAC 2007, arXiv:0712.2201, 2007.

[27] M. Uchiyama. Two-species asymmetric simple exclusion process with open boundaries. Chaos Solitons Fractals, 35(2):398-407, 2008.

[28] M. Uchiyama, T. Sasamoto, and M. Wadati. Asymmetric simple exclusion process with open boundaries and Askey-Wilson polynomials. J. Phys. A, 37(18):4985-5002, 2004.

[29] G. Viennot. Une théorie combinatoire des polynômes orthogonaux. Lecture Notes UQAM, Publication du LACIM, page 219p., 1984. 